\title{
Research on the Industrial Robot Control System Based on the Ontology and Web Service
}

\author{
Huili Xia ${ }^{1}$ Yaohe Liu' ${ }^{1}$ Tingxin Song ${ }^{1,2}$ Baohua Tan ${ }^{1}$ \\ ${ }^{1}$ Hubei University of Technology, Wuhan 430068, P. R. China \\ ${ }^{2}$ Tsinghua University, Beijing 100084, P. R. China
}

\begin{abstract}
In this paper we briefly present the concepts of ontology and Web Service, analyze the advantages that introduce them into robot control system. This research constructs the ontologies related with the control parameters of machine manipulator, designs a realization model and the corresponding services composition framework for the semantic Web Service based on ontology. Experimental results show that the approach improves the versatility of the various robot control systems, realizes the asynchronous communication within the clients and Web Service composites, thus enhances the real-time implementation of remote industrial robot control system as well as displays the information validity.
\end{abstract}

Keywords: Ontology, Web Service, Services composition framework, Asynchronous communication, Remote industrial robot control system

\section{Introduction}

With the rapid development of intelligent control technology such as neural network, annealing algorithm, machine learning and reasoning, the research of industrial remote robot control via internet [1] has been making a bigger progress.

Nowadays, the research and development in the algorithm of robot control system have been becoming more mature. During the procedure of the robot control, different control algorithms have provided necessary guarantees for the control system. Robust, mature and easy-to-use tools play crucial roles for the easy adoption of any new technology. However, diversification and complication of different control parameters and workpiece information will come up with many problems for the control algorithm implement. It mainly manifests that in the different control system, the expression of parameter resources exists the feature as source broad, not unified, large quantities etc. Consequently, the knowledge denotation becomes isolate, irrelative and absent that brings problems in the realization and development of various robot control system, reducing the system versatility greatly. To resolve these problems, it is very essentially and urgent to construct the ontologies related with the control parameters of industrial robot, which is applied in the semantic system that the machine could understand in the certain domain.

The combination of web and robot control technology gave birth to the concept of Web Service based remote robot [2]. Recently, how to semanticize the Web Service which integrates into control parameters ontologies and adapts to the real industrial robot control system has turned into one of focal problems with universal attention in enterprises and academic circles.

In this paper, we constructed an ontologies related with the robot which is reconfigurable, reusable and expandable. Taking the parameters semantic into account, we provided automatic composite Web service framework based on the ontologies and realized asynchronous communication within the clients and Web Service composites. Finally, match process begin choose the sub-service including proper parameter semantic from the available service list. According to the executed program in match process, the composite Web Service could correctly select and transfer the relevant sub-service. In the end of the paper, the concrete realization of intellectualization industrial remote robot control system has been discussed further.

\section{Construction of ontology for the manipulator parameters}

\subsection{The ontology concepts}

Ontology [3] is a term borrowed from philosophy which has not a strict unified definition yet. It is an important emerging discipline that has the great potential to improve information organization, management and understanding. Based on the formal 
notion of "conceptualization", which is accepted by many people with the ontology definition in the computer field, is: "Ontology is an explicit semantic representation of data, accompanied with domain theories [4]". In the knowledge sharing context, ontology is specified in the form of definitions of representational vocabulary. Shared conceptualizations include conceptual frameworks for communicating modeling domain knowledge; content-specific protocols among inter-operating agents; and agreements about representation of particular domain theories. The goal of the ontology is that the content of information shall be described in a machineunderstandable way, not only read by human. Because Ontology aims at consensual domain knowledge, the importance of domain ontologies [5] is widely recognized. As the term refers to the shared understanding of some domains, Ontology is often conceived as a set of concepts, relations, functions, axioms and instances. It's a shared view of a domain conceptualization - taxonomy which can build a "cross bridge" between the needs and the description for the client resources.

Recently, ontology theory has already aroused widely attention among domestic academic circles. Many experts and scholars are researching on the ontology engineering, expression, transformation, integration and application based on their professional field. In our research, as for the industrial robot control system domain [6], the focal task is how to express, organize and share various knowledge effectively for building the modularized, knowledge-based and personalized ontologies.

\subsection{The construct process of ontology for the manipulator parameters}

As we know, one of the barriers to a wider adoption of ontology-based web service technology is the lack of tools for creating semantic specification. Once the ontology is defined, it can be used to represent information in the real world independently. Thus creating ontologies is an iterative process that there is no one linear approach for developing ontologies with any tool. In this paper, we take the machine manipulator as the example and put forward a prototype model for structuring process of domain ontology, which consult the idea and experience of software engineering.

The basic steps of the process are described as in Figure 1.

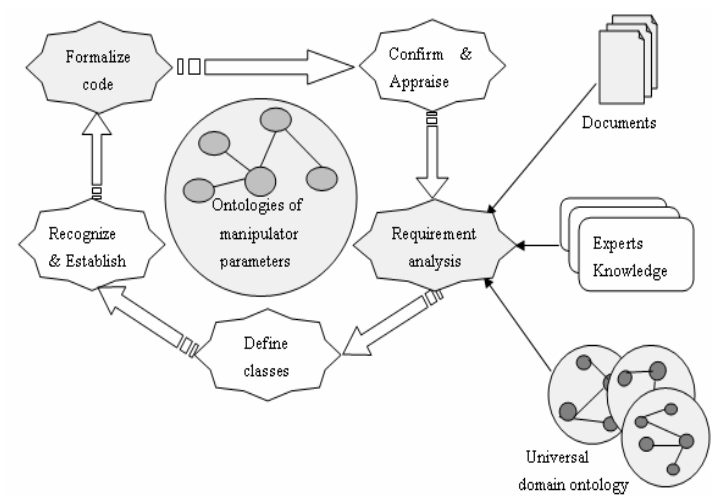

Fig. 1: The prototype model of the construction of domain ontology for the manipulator parameters.

The prototype model is an annular structure. It starts out by determining the scope in defining all the different concepts from the universal domain ontology. After designating the robot control system as the domain fundamental ontology, we should collect the professional knowledge in the mechanical domain so as to ensure the requirements Analysis.

The procedure of the proposed prototype model is described below:

- Requirements Analysis: Similar to the software developing method, we should capture information from its application scope, purpose as well as users from the beginning of constructing ontology, and the final goal of domain ontology is for the machine understanding.

- Define classes: Carrying on the detailed analyze and classifying the concept which is a collection of elements with similar properties from the requirement analysis. The properties would describe various features and attributes of the class.

- Recognize terms and establish the ontology framework: Evaluating the importance of every concept and selecting the key terms to removing the concepts that are unnecessary or exceed the domain scope. In this stage, the task is complete the construction of target ontology structure which describes the concepts in the domain and also the relationships that hold between those concepts.

- Formalize code: After making evaluation, validation and refinement of the terms in the certain domain, we should formalize the good terms in codes in order to let the information have the machine intelligibility. Thus the implicit semantic of the relational model must be mapped into explicit ontological structures. In the procedure of constructing ontology, it 
would use the "ontology reconfigurable" features and "ontology mapping" technologies.

- Confirmation and appraisal: The objective of this task is appraising whether the existing ontology is conceptually rich enough to represent relational schema completely and confirm whether all relational entities are mapped into corresponding ontological entities.

Because the domain knowledge is infinite, the domain boundary is fuzzy and the domains are overlap, we should continue communicating with professional expert after establishing the primary edition of certain domain ontology [7].

In order to unceasingly enrich the contents of domain ontology, we can use the ontology evolution method to keeping discovering the new knowledge.

Once the ontology is created, we need migrate the data, which task is the creation of ontological instances based on the relational database.

In this paper, we have built the domain ontology of manipulator based on our approach following the overall prototype model given above. In order to best facilitate these tasks, we construct the ontologies in the Protégé-OWL Ontology Editor. Protégé [8] is an open-source ontology development environment developed at Stanford Medical Informatics. The OWL plug-in is a major extension of Protégé providing interfaces to Description Logics Reasoners i.e. Racer and allowing accessing other services.

Below is the domain ontologies of manipulator Parameters.

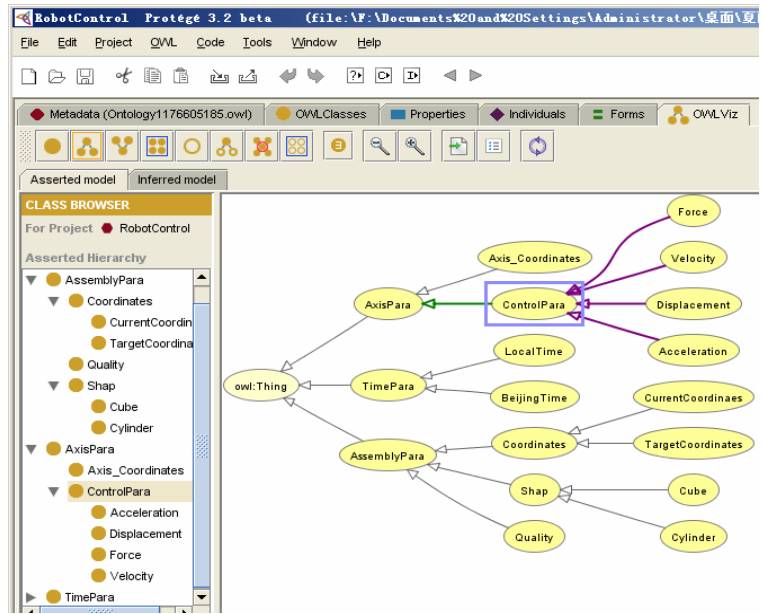

Fig. 2: The snapshot of ontology description for the manipulator parameters in Protégé.

The ontology and the corresponding relations within the ontologies are shown. We have constructed several ontology classes in Protégé, namely:
- AxisPara. Including coordinates and control parameters (e.g. force, acceleration, velocity, displacement ) related with the axis.

- AssemblyPara. Including quality, shape and coordinates of the workpiece.

- TimePara. Including local time and beijing time.

\section{The construction of semantic Web Service model based on ontology}

\subsection{The Web Service architecture}

Web Service is a technology that allows applications to communicate with each other in a platform and programming-language-independent manner. The main function of industrial robot control system based on Web Service [9] is to divide the whole control system into several self-configurable intelligent systems that are separated in different physical addresses and locations. While Web Service allows all these dynamic features to compose multiple services into applications, you still have to build the services first. Each service translates and analyzes the message according to its local implementation and environment. These sub-systems can resolve the corresponding tasks independently and intercommunicate in the common ground for the fulfillment of whole system simultaneously. Above all, the most centralized question manifests in the system structure design and the corresponding cooperation mechanism. The single Web Service, which is dynamic, always occupies in multiple Web Service system environments, sometimes needs the Web Service to make the rapid response for adapting to the environment and processing Web Service contradiction and deploying the coordination meanwhile. Eventually, the Web Services plan its behavior, transmit the news, disseminate the robot motion logic information and make the decision.

Figure 3 gives out the Web Service architecture.

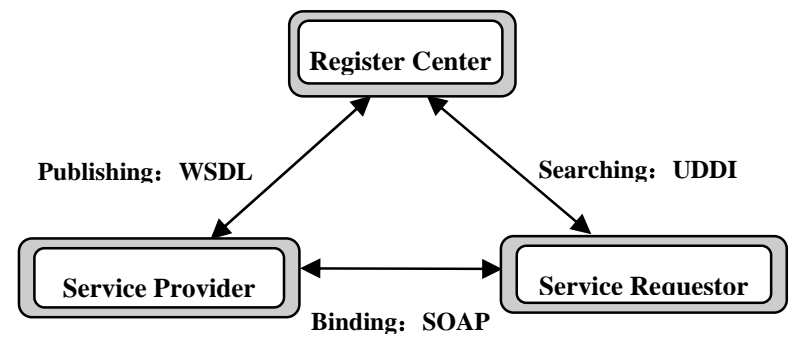

Fig. 3: The Web Service architecture. 
A Web Service provider developed his own applications and offers services on the Web in WSDL (Web Services Description Language).The registry provides standardized description facilities to describe all the corresponding information about a particular service in UDDI (Universal Description, Discovery, and Integration), the registry publishes and locates services. Then the service requestor uses the SOAP (Simple Object Access Protocol) description to create a binding for her application to be able to invoke or interact with the Web Service implementation [10].

In the area of industrial robot control system, we provide semantic Web Service models which integrate the manipulator parameters ontology into the Web service architecture.

\subsection{Semantic Web Service model}

Based on the manipulator parameters ontology, the semantic Web Service model (in Figure 4) fulfills the corresponding composite Web Service function with

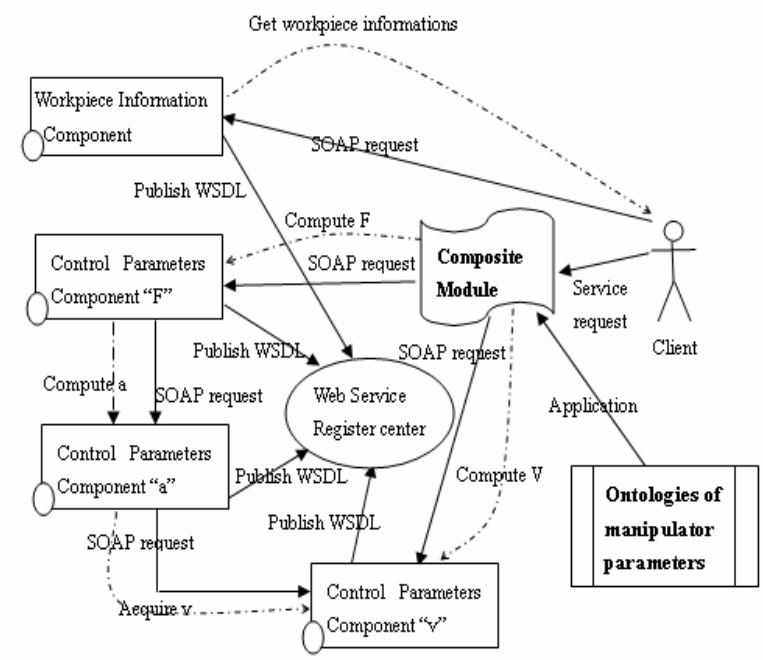

the service match according to the request of client.

Fig. 4: The semantic Web Service model based on the ontology of the manipulator parameters.

The invoked procedure of Web service operation in four main steps:

- Registering all Web Service components in the registry for querying.

- The requestor calls the components of workpiece information asynchronously in the SOAP and receives the workpiece data.

- The requestor sends the service requests related with the operation of manipulator.

- Invoking the service match and composite module to realize the function of calling service module asynchronously. In our case, we utilize the manipulator ontologies to automatic match the composite scheme that meets the control function based on the algorithm of robot control system.

For example: to realize the planning and composition of the axis motion in the multidimensions, a nonlinear differential equation[11] formulates the manipulator dynamic state which is (1).

$$
M(q) \ddot{q}+C(q, \dot{q}) \dot{q}+G(q)+F(q, \dot{q})=\tau
$$

In the procedure of computing this equation, we need use the ontologies. At first, the system should gain workpiece information and the axes state parameters of every arms; then, distribute the parameters computation into the corresponding Web Service modules with the reasoning Web Service composite module according to the logical attaching relation which has defined in the ontologies. Thus the control system completes the asynchronous operation of manipulator dynamic equation and motion control.

\subsection{The Web Service architecture}

The purpose of service composite is to realize the automated processing of controlling the robot motion flow. And the semantic of parameters is defined in the ontologies [12]. How to realize automatic match of Web Service based on semantic is a difficulty. The exterior view of the service composite is showed in figure 5 .

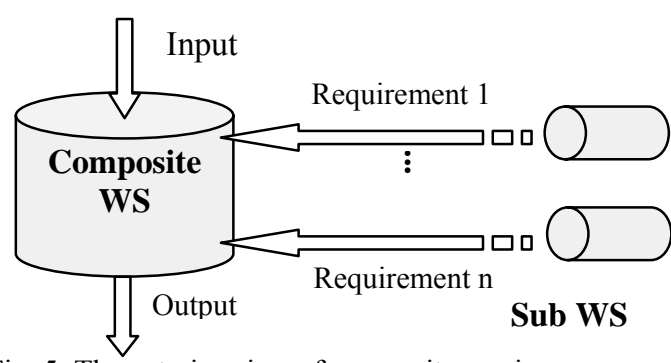

Fig. 5: The exterior view of composite service.

There is composite service on the left, it accepts the input information of service requestor and acquires his demands from several sub-services in the procedure of completing composite function, and returns the output result to clients. While observing the composite service from the exterior view, it must actively send out the demand to the outside beside self-operation, so it can be regarded as coordinator of resource. Service.

Figure 6 gives out the Interior View of Composite

In generally, in the interior view (in Figure 6), the composite service has been divided into two procedures to finish the external function. 
Top layer: Crawler Process

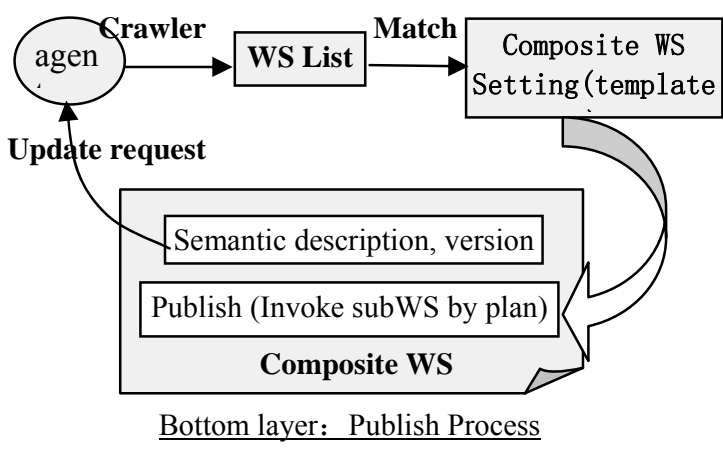

Fig. 6: The interior view of composite service.

One procedure is the match process. The composite services find the semantic services related with the match according to the users' requests. This is the searching period of the service (Crawler). Then the search results of every service (recorded in WS List) should be analyzed to compare their semantics with the service requirements. The parameter semantics of the services (Ontology) are loaded to OWL reasoned to inference to find whether the searching results meet users' needs. This is the matching period of service (Matcher). At last, the sub-services which meet the needs should be ordered, and the calling information and version information should be recorded to the configuration file. This is the services' recording period of service (WS Setting).

Another is the publishing process. This process is the operating period of service. When receiving a user's invoking, the composite service reads the configuration file firstly, then checks and invokes the referring sub-services in configuration file. In the end, the composite service calculates the results of subservices to return to the users. Meanwhile, the composite service and its' controlling information directing on semantic description and version will be forwarded too.

In our research, the system can be realized by ASP.NET in C\# to develop the Web interface that client can call the Web forms by the Web explorer in the HTTP protocol. The final goal of the Crawler is discovering the semantic service related with match process according to the client requests, which is based on the control operation of industrial robot.

Because a service provides many methods, we declare one method as:

[WebMethod]

public string svGetServiceDescription( )

\{

return;
@,"http://localhost/Webservice/WebSite1/Get Contr-olPara.xml";

\}

Explanation: Match explorer try to call the svGetServiceDescription( ) of every service in the operation of Crawler. If succeed, load the match result in the Configuration files with register.xml; on the contrary, it indicates this service should not be the semantic service which we expected.

The system adopts the method of service match to implement the automatic deploy in the Web Service. In our research, we use the Pellet Reasoner to operate the analyzing and reasoning of semantic during the match process which is realized in the Eclipse 3.2 platform in Java, and then load the result into the composite setting in XML(Extended Makeup language). Thus it will complete the opration of executing composite srevice and realize the whole control flow.

\section{Conclusions and further research}

The approach described in this paper has shown that ontologies can provide the structure and mechanism that enable an intelligent system which can apply in the control system of remote industrial robot based on the semantic Web Service. We also constructed the Ontology for the manipulator parameters through OWL and RDF (Resource Description Framework) and provided a composite web service framework.

In the future work, we will develop the ontology to include a broader range of abstract concepts that could be exploited by the different robot control system .Along with development of the Ontologies Involved, we will apply Semantic Web Service to the architecture. Because of the semantically description of the Web Services, the Web Services will be able to be semantically discovered, semantically executed and semantically composed. It will be a big challenge to resolve these in our future work.

\section{Acknowledgement}

This work is partially supported by National Nature Science Foundation of Hubei Provincial Department of Education in China (Grant No. 2003Z002).

\section{References}

[1] P. Hu, The study and realization of an open structure robot control system, Wuhan University of Science, 2006. 
[2] L. Lu and B. L. Shi, Research on remote robot control system based on web, ROBOT 23(2):152155, 2001.

[3] M. Alexander and S. Steffen, Ontology learning for the Semantic Web, IEEE Intelligent Systems (Special Issue on Semantic Web) , 16(2):122148, 2001.

[4] S. Vijayan and C. S. Veda, The role of domain ontologies in database design: An ontology management and conceptual modeling environment, ACM Transactions on Database Systems, 31(3):1065-1068, 2006.

[5] N. Roberto and V. Paola, Learning domain ontologies from document warehouses and dedicated web sites, Computational Linguistics, 30(2):151-168, 2004.

[6] M. Rogan and W. Mary-Anne, Ontology based object categorization for robots, Conferences in Research and Practice in Information Technology Series, 58:65-80, 2005.

[7] M. H. Yang, L. Q. Qian, W. Y. Zhao, Research on merging ontology to generate domain-specific ontology, Computer Engineering, 2006.

[8] Gennari, J. Musen, M. Fergerson, R. Grosso, W. Crubézy, M. Eriksson and H. Noy, The evolution of Protégé-2000: An environment for knowledgebased systems development, International Journal of Human-Computer Studies, 58(1):89-106, 2003.

[9] Z.X. Yang, Study of robot control system based on Web Service, Hubei University of Technology, 2005.

[10] N. Gu, J.M. Liu and X. L. Chai, The principle and realization of Web Services, Beijing: china machine press, 2006.

[11]L.B. Ma , Research of telerobotics control system on the internet and key technology, Chongqing University, 2003.

[12] W.N. Tang and F.Y. Xu, Supply chain knowledge integration based on ontology and semantic Web Services, Computer Engineering, 32(24):167-169, 2006. 